\title{
Time to hospital admission for acute stroke: an observational study
}

STROKE AFFECTS AN ESTIMATED 40000 Australians annually, ${ }^{1}$ with an associated cost of A $\$ 1.3-\$ 1.7$ billion per year. ${ }^{2,3}$ Thrombolytic therapy has been shown to improve neurological outcome of acute ischaemic stroke if given within three hours of symptom onset. ${ }^{4}$

This new emphasis on acute management of stroke has widespread implications. As thrombolysis has many contraindications and exclusion criteria (eg, haemorrhagic stroke, coma and severe deficit), ${ }^{4}$ the diagnosis of ischaemic stroke must be confirmed, and clinical evaluation and investigations completed within the three-hour "window" after symptom onset. Assuming the time required for investigations, hospital admission and obtaining consent can be reduced to about an hour, patients must arrive at hospital within two hours of symptom onset if they are to be eligible for thrombolysis. Both health professionals and the general public need to be more aware of stroke as a medical emergency to facilitate this early arrival.

Indeed, previous studies have shown that the single most important factor in determining eligibility for thrombolysis is time to admission. ${ }^{5}$ Although factors associated with this time have been studied overseas, there are no comparable findings for Australia. Given the large distances between centres where thrombolytic therapy might be given in this country, it may not be appropriate to extrapolate findings from more densely populated parts of the world.

We therefore undertook a prospective study of time to admission for patients with suspected acute stroke at the Royal Adelaide Hospital (SA), and the factors associated with this time, as well as final

\section{ABSTRACT}

Objectives: To determine the time from symptom onset to hospital admission of patients with suspected acute stroke, final diagnoses and patient eligibility for thrombolytic therapy.

Design: Hospital-based, prospective, observational study.

Setting: Royal Adelaide Hospital Stroke Unit, South Australia.

Patients: All patients admitted to the unit with suspected acute stroke over 11 months (11 April to 10 October 2000 and 20 August 2001 to 19 January 2002).

Main outcome measures: Time from symptom onset to admission; final diagnosis.

Results: Of 284 patients admitted, 39 (14\%) had diagnoses other than stroke (including eight with transient ischaemic attacks), 42 (15\%) had haemorrhagic stroke and $203(71 \%)$ had ischaemic stroke. Median time to admission after symptom onset was 6 hours (range, 30 min to 13 days), with 100 patients admitted within 3 hours of symptom onset (35\%), and 80 within 2 hours (28\%). Thirty-seven patients $(13 \%)$ could have been considered for thrombolysis (diagnosis of non-severe but disabling ischaemic stroke and admission time $<3$ hours). Location at stroke onset was the only independent predictor of time to admission.

Conclusions: Most patients with stroke do not present urgently to the emergency department, rendering them less likely to be considered for thrombolytic therapy.

MJA 2003; 178: 329--331

diagnoses and proportion of patients eligible for thrombolysis.

\section{METHODS}

This was a hospital-based, observational study at the Royal Adelaide Hospital Stroke Unit. This hospital serves a local population within the metropolitan area of Adelaide of about 250000 and is a tertiary referral centre for community hospitals throughout South Australia (population, 1.8 million), the Northern Territory, south-western New South Wales and western Victoria. The Stroke Unit has a multidisciplinary team and admits all patients with a clinical diag-

For editorial comment, see page 309; see also pages 318,324 and 333 .

Department of Neurology, Royal Adelaide Hospital, and Department of Medicine, University of Adelaide, Adelaide, SA.

Simon A Broadley, PhD, MRCP, Advanced Trainee;

Philip D Thompson, PhD, FRACP, Professor of Neurology.

Reprints will not be available from the authors. Correspondence: Professor Philip D Thompson,

Department of Neurology, Royal Adelaide Hospital, Adelaide, SA 5000.

philip.thompson@adelaide.edu.au nosis of acute stroke, regardless of age or severity (except those requiring neurosurgical intervention). The study met the ethical requirements of the Royal Adelaide Hospital Research Ethics Committee.

\section{Data source and extraction}

Information was collected prospectively on all patients admitted to the Stroke Unit during two periods, totalling 11 months (11 April to 10 October 2000 and 20 August 2001 to 19 January 2002).

Time and patient location at symptom onset were ascertained from patients or relatives, along with demographic and basic clinical details. For patients who woke with symptoms, time of onset was taken as the time of waking. For those who were admitted unconscious after being found collapsed, time of onset was taken as the last time they were witnessed to be well. Patients who suffered stroke while inpatients were excluded from this study.

Time to admission was calculated using the arrival time routinely logged in 


\section{1: Final diagnoses in 284 patients admitted with suspected stroke}

\begin{tabular}{|c|c|}
\hline Stroke & $245(86 \%)$ \\
\hline Infarction & $203(71 \%)$ \\
\hline Lacunar & 56 \\
\hline Embolism & 38 \\
\hline Large artery occlusion & 89 \\
\hline Carotid artery stenosis & 10 \\
\hline $\begin{array}{l}\text { Carotid/vertebral artery } \\
\text { dissection }\end{array}$ & 10 \\
\hline Haemorrhage & $42(15 \%)$ \\
\hline Intracerebral & 39 \\
\hline $\begin{array}{l}\text { Venous infarction with } \\
\text { haemorrhage }\end{array}$ & 3 \\
\hline Non-stroke & $39(14 \%)$ \\
\hline Transient ischaemic attack & 8 \\
\hline Migraine & 6 \\
\hline Seizure disorder & 5 \\
\hline Conversion disorder & 4 \\
\hline Dementia & 3 \\
\hline Intoxication/overdose & 2 \\
\hline Peripheral nerve lesion & 2 \\
\hline $\begin{array}{l}\text { Previous stroke with intercurrent } \\
\text { illness }\end{array}$ & 2 \\
\hline Space-occupying lesion & 2 \\
\hline Other* & 5 \\
\hline
\end{tabular}

* Single cases of idiopathic cerebellar atrophy, adenocarcinoma-associated opsoclonus-myoclonus, hydrocephalus,

vestibular neuronitis and multiple sclerosis.

emergency department records. Location of the patient at stroke onset was denoted by postcode and categorised as Adelaide, regional-rural South Australia or interstate, and according to the Accessibility/Remoteness Index of Australia (ARIA). ${ }^{6}$

A stroke syndrome was defined as the rapid onset of a neurological deficit, conforming to a vascular territory and without other evident cause. Stroke symptoms typically last over 24 hours. For practical purposes, a decision whether a neurological event is a stroke must be made much sooner than the definition implies, and some vascular events turn out to be transient ischaemic attacks in which symptoms resolve within 24 hours.

Strokes were further classified according to size, location and nature of the vascular event, based on clinical and investigation findings. All patients underwent neuroimaging with either

\section{2: Eligibility for thrombolysis of 100 patients with suspected stroke admitted within three hours of symptom onset}

\begin{tabular}{|c|c|c|}
\hline & \multicolumn{2}{|c|}{ Time from symptom onset to admission } \\
\hline & $<2 \mathrm{~h}(n=80)$ & $<3 \mathrm{~h}(n=100)$ \\
\hline Excluded from thrombolysis (\% of all patients*) & $51(18 \%)$ & $63(22 \%)$ \\
\hline Non-stroke & 11 & 16 \\
\hline Haemorrhagic stroke & 14 & 19 \\
\hline Severe stroke ${ }^{\dagger}$ & 21 & 23 \\
\hline Non-disabling stroke & 5 & 5 \\
\hline $\begin{array}{l}\text { Eligible for consideration of thrombolysis } \\
\text { (\% of all patients) }\end{array}$ & $29(9 \%)$ & 37 (13\%) \\
\hline
\end{tabular}

computed tomography (CT) or magnetic resonance imaging (MRI). Stroke severity was assessed using the modified Barthel Index (range, 0-100, where $0=$ total incapacity and $100=$ independent $)^{7}$ within three days of admission.

\section{Data analysis}

Odds ratios with 95\% CIs were used to assess factors influencing admission within three hours. Multiple logistic regression analysis was used to examine independent associations and to calculate adjusted odds ratios.

\section{RESULTS}

During the study period, 284 patients were admitted with a suspected diagnosis of acute stroke. Final diagnoses and stroke subtypes are summarised in Box 1. Stroke was confirmed in 245 patients (86\%), while 39 patients (14\%) had other diagnoses, including eight with transient ischaemic attacks. Most strokes were ischaemic $(203 ; 83 \%)$.

Mean age of the 245 patients with confirmed stroke was 72 years (range, 20-100 years), and 138 (56\%) were male. Twenty-five patients (10\%) were unconscious on admission, and the median modified Barthel Index was 51 (range, 0-100).

\section{Admission time}

Median time from symptom onset to admission for all suspected strokes was 6 hours (range, 30 minutes to 13 days). One hundred patients $(35 \%$ of all patients) were admitted within three hours of symptom onset, and $80(28 \%)$ within two hours. Assessment and investigation reduced the number of patients who could have been considered for thrombolysis to 37 of those admitted within three hours (13\% of all patients) and 26 of those admitted within two hours (9\%) (Box 2).

\section{Factors associated with admission time}

Time to admission was found to be shorter for patients with more severe strokes (modified Barthel Index $<50$, large lesions on CT or MRI), who were unconscious on admission and lived in an area of high accessibility (ARIA score <1.84) (not shown). However, after adjustment for confounding factors, the only independent predictor of earlier admission was living in an area of high accessibility (Box 3).

The effect of geographic location at the onset of stroke on admission time is shown in Box 4. Of the 184 patients admitted more than three hours after symptom onset, 76 lived outside metropolitan Adelaide.

There was no significant difference in the time to admission between the two periods of study.

\section{DISCUSSION}

In this study of 284 patients admitted to hospital with suspected stroke, 35\% were admitted within three hours of symptom onset, and $28 \%$ within two hours. These values fall within the range found in North America, where $27 \%-50 \%$ of stroke patients were admitted within three hours of symptom onset. ${ }^{5,8,9}$ 


\section{3: Characteristics of $\mathbf{2 4 5}$ patients with stroke according to time from symptom onset to admission}

\begin{tabular}{|c|c|c|c|}
\hline & \multicolumn{2}{|c|}{ Time from symptom onset to admission } & \multirow{2}{*}{$\begin{array}{l}\text { Adjusted odds } \\
\text { ratio }(95 \% \mathrm{Cl})^{*}\end{array}$} \\
\hline & $<3 h(n=84)$ & $\geqslant 3 \mathrm{~h}(n=161)$ & \\
\hline Age $>59$ years & $72(86 \%)$ & $118(63 \%)$ & $1.51(0.70-3.25)$ \\
\hline Female & $40(48 \%)$ & $67(42 \%)$ & $0.93(0.52-1.67)$ \\
\hline Highly accessible locality ${ }^{\dagger}$ & $77(92 \%)$ & $114(71 \%)$ & $5.22(2.14-12.75)$ \\
\hline Unconscious on admission & $14(17 \%)$ & $11(7 \%)$ & $1.49(0.54-4.05)$ \\
\hline Dysphasia & $23(27 \%)$ & $37(23 \%)$ & $1.05(0.54-2.06)$ \\
\hline Modified Barthel Index $<50$ & $52(62 \%)$ & $71(44 \%)$ & $1.62(0.84-3.14)$ \\
\hline Primary haemorrhage & $19(23 \%)$ & $20(12 \%)$ & $1.51(0.67-3.37)$ \\
\hline Lesion $>3 \mathrm{~cm}$ on $\mathrm{CT} / \mathrm{MRI}$ & $42(50 \%)$ & $52(32 \%)$ & $1.69(0.87-3.26)$ \\
\hline
\end{tabular}

$\mathrm{CT}=$ computed tomography. $\mathrm{MRI}=$ magnetic resonance imaging.

${ }^{*}$ All variables included in model.

† Defined as score on Accessibility/Remoteness Index of Australia (ARIA) scale $<1.84$ (corresponding to unrestricted access to services). ${ }^{6}$

After assessment and investigation, $14 \%$ of patients in our study were diagnosed with conditions other than stroke - a figure comparable with previous studies. ${ }^{10}$ In typical cases of stroke, there may be little diagnostic uncertainty. However, expert assessment and imaging is still necessary to confirm the diagnosis and, most importantly, to exclude intracerebral haemorrhage and define the stages of ischaemic stroke if thrombolysis is to be considered. Accordingly, management of acute thrombolytic stroke should be restricted to specialist units where appropriate assessment, including imaging and monitoring, is readily available.

We also found that, after investigation, the number of patients eligible for consideration of thrombolytic therapy ${ }^{4}$ fell to 37 among those presenting within 3 hours of symptom onset $(13 \%$ of all patients) and 29 among those presenting within 2 hours ( $9 \%$ of all patients). These figures may still be overestimates, as they do not take into account other standard exclusion criteria for thrombolysis, including previous use of anticoagulation therapy, recent surgery, haematological disorders, severe hypertension and seizures. ${ }^{4}$ Furthermore, we took time of onset as time of waking for those who developed symptoms while asleep. Changing this criterion to time of falling sleep, as in other studies, might further reduce the numbers who could be considered for thrombolysis.

Almost three-quarters of our patients $(72 \%)$ did not present urgently (within two hours of symptom onset) to the hospital. Delayed admission can be explained in part by remoteness from hospital, but only in a minority of patients. Factors shown to be associated with delayed admission in other studies include uncertain time of symptom onset, ${ }^{8,9}$ contact with a primary care physician, transportation by means other than an ambulance, and patient failure to perceive danger. ${ }^{8,9,11,12}$ Loss of consciousness and seizures at onset are associated with shorter admission time. ${ }^{8}$ Knowledge of the nature and significance of warning symptoms and signs of acute stroke is poor in the urban Australian population, ${ }^{13}$ and failure to recognise the significance of stroke symptoms was a significant cause of delay in presenting to hospital in a recent study in New South Wales. ${ }^{14}$

These findings demonstrate a need for public education to optimise the use of new interventional therapies in stroke. Further study is needed in Australia to determine the origin of referral to hospital, mode of transport and priority of triage on arrival at hospital.

We conclude that the successful implementation of thrombolysis for acute stroke will require changes in the organisation of emergency services and hospital departments, as well as in attitudes of the general public toward stroke. The implications for resource allocation and the provision of 24-hour acute neurological assessment and interventional services also need to be evaluated.

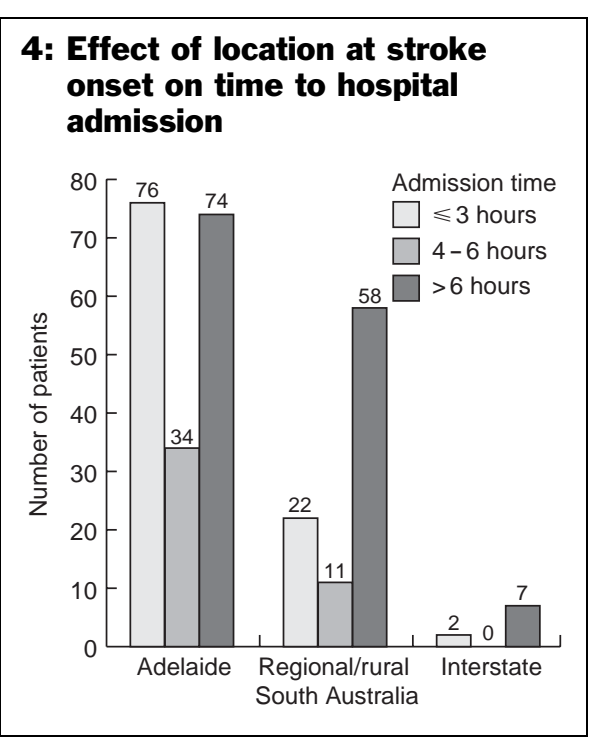

COMPETING INTERESTS

None identified.

\section{ACKNOWLEDGEMENTS}

We are grateful to Dr Emma Whitham, Dr Ramon Pathi, Dr Derek Yiu, Dr Edward Teo, Dr Vicky Chong, Dr David Cardone and Dr Basil Alexander (Stroke Unit, Royal Adelaide Hospital, SA) for assisting with data collection.

\section{REFERENCES}

1. Bonita R, Anderson C, Broad J, et al. Stroke incidence and case fatality in Australasia. Comparison of the Auckland and Perth population-based registers. Stroke 1994; 25: 552-557.

2. Hankey G. Transient ischaemic attacks and stroke. Med J Aust 2000; 172: 394-400.

3. Dewey H, Thrift A, Mihalopoulos C, et al. Cost of stroke in Australia from a societal perspective: results from the North East Melbourne Stroke Incidence Study (NEMESIS). Stroke 2001; 32: 2409-2416.

4. The National Institute of Neurological Disorders and Stroke rt-PA Stroke Study Group. Tissue plasminogen activator for acute ischaemic stroke. $N$ Engl J Med 1995; 333: 1581-1587.

5. Barber P, Zhang J, Demchuk A, et al. Why are stroke patients excluded from TPA therapy? An analysis of patient eligibility. Neurology 2001; 56: 1015-1020.

6. Dunne L, Hugo G, Bamford E, et al. Measuring remoteness: accessibility/remoteness index of Australia (ARIA). Canberra: Commonwealth Department of Health and Aged Care, 2001: 1-25. Available at http:// www.health.gov.au/ari/aria.htm (accessed Sep 2002).

7. Shah S, Vanclay F, Cooper B. Improving the sensitivity of the Barthel Index for stroke rehabilitation. J Clin Epidemiol 1989; 42: 703-709.

8. Smith M, Doliszny K, Shahar E, et al. Delayed hospital arrival for acute ischaemic stroke: the Minnesota Stroke Survey. Ann Intern Med 1998; 129: 190-196.

9. Lacy C, Suh D, Bueno M, Kostis J. Delay in presentation and evaluation for acute stroke: Stroke Time Registry for Outcomes Knowledge and Epidemiology (S.T.R.O.K.E.). Stroke 2001; 32: 63-69

10. Norris JW, Hachinski VC. Misdiagnosis of stroke. Lancet 1982; 1: 328-331

11. Salisbury H, Banks B, Footitt D, et al. Delay in presentation of patients with acute stroke to hospital in Oxford. QJM 1998; 91: 635-640.

12. Srivastava A, Prasad K. A study of factors delaying hospital arrival of patients with acute stroke. Neurol India 2001; 49: 272-276.

13. Yoon S, Heller R, Levi C, et al. Knowledge of stroke risk factors, warning symptoms, and treatment among an Australian urban population. Stroke 2001; 32: 1926-1930.

14. Yoon S, Byles J. Perceptions of stroke in the general public and patients with stroke: a qualitative study. BMJ 2002; 324: 1065-1068.

(Received 11 Mar 2002, accepted 29 Sep 2002) 